\title{
Toxocarosis y colecistitis aguda alitiásica ¿consecuencia o coincidencia?
}

\author{
Alexis Strickler, Natalia Vásquez, Leonardo Maggi, Juvenal Hernández y Ximena Hidalgo
}

\footnotetext{
Universidad San Sebastián, Puerto Montt. Departamento de Pediatría (AS, NV, LM) Hospital Dr. Eduardo Schütz Schroeder, Puerto Montt, Chile. Servicio de Pediatría $(\mathrm{XH})$ Servicio de Imagenología $(J H)$

Recibido: 4 de septiembre de 2015 Aceptado: 26 de abril de 2016

Correspondencia a: Alexis Strickler alexisstrickler@gmail.com
}

\section{Toxocarosis and acalculous acute cholecystitis. Consequence or coincidence?}

Human toxocarosis is a chronic larval parasitosis listed as one of the five most important neglected diseases by the CDC. The larvae can spread systemically and migrate to different tissues including liver and gallbladder. Acalculous acute cholecystitis (AAC) is a rare disease in children. The diagnosis is based on clinical parameters and imaging criteria. It has been reported in relation to sepsis, shock, trauma, burns, severe systemic diseases, congenital anomalies, infections and also in healthy children. We report a pediatric case of toxocarosis, with clinical symptoms and imaging criteria compatible with AAC treated medically, and discuss the relationship between toxocarosis and AAC based on published evidence.

Key words: Toxocarosis, acalculous acute cholecystitis, pediatrics, albendazole.

Palabras clave: Toxocarosis, colecistitis aguda acalculosa, colecistitis aguda alitiásica, pediatría, albendazol.

\section{Introducción}

$\mathrm{E}$ 1 dolor abdominal es un motivo frecuente de consulta en unidades de emergencia, atención primaria y gastroenterología pediátrica. Pese a su inespecificidad, la descripción detallada del síntoma, su relación temporal, junto con los antecedentes mórbidos personales, familiares y epidemiológicos, permiten seleccionar los diagnósticos más probables, dentro de un amplio espectro de diagnóstico diferencial, que incluyen las parasitosis y las enfermedades de la vesícula biliar.

La toxocarosis es una infección parasitaria larval sistémica, de distribución mundial, observada preferentemente en la niñez, con alta prevalencia en Latinoamérica, causada por la ingestión de huevos embrionados de Toxocara canis en la mayor parte de los casos o Toxocara catis. Los síntomas clínicos dependen de los órganos invadidos y puede cursar en forma asintomática, compromiso local o generalizado (larva migrans visceral).

La colecistitis aguda (CA) es una enfermedad inflamatoria aguda de la vesícula biliar. En niños, la CA es infrecuente y puede manifestarse como CA litiásica, o CA acalculosa (CAA). En comparación con adultos, en que sólo 2-17\% de los casos de las CA son no litiásicas, $30-50 \%$ de los casos pediátricos de CA son acalculosas. El diagnóstico clínico se sustenta en los síntomas clínicos y los hallazgos imagenológicos. La causa más frecuente es infecciosa, incluyendo los parásitos.

Presentamos el caso clínico de un niño de 8 años, con infección por Toxocara spp. demostrada, quien presentó síntomas clínicos, signos sonográficos y tomográficos compatibles con CAA. Además se revisa la evidencia y se discute la validez del diagnóstico.

\section{Caso clínico}

Escolar de sexo masculino, con ocho años de edad, obeso (IMC 20,1), con residencia urbana. Tenía contacto con una mascota (perro) vacunada, pero sin control periódico. Tenía antecedentes de un episodio de enteroparasitosis (Entamoeba coli) a los 4 años de edad, tratado con mebendazol durante tres días, además de ingestión habitual de salmón ahumado, con una última ingesta tres meses antes, sin molestias inmediatas. Padres con sobrepeso y sin antecedente de colecistitis/colelitiasis en familiares directos.

Consultó en la unidad de emergencia hospitalaria por dolor abdominal epigástrico postprandial intenso, con carácter cólico, asociado a náuseas recurrentes de 15 días de evolución, sin fiebre, ictericia, diarrea, acolia ni coluria. En el examen físico segmentario sólo destacaba una sensibilidad abdominal difusa de predominio epigástrico, sin visceromegalia. Ingresó con el diagnóstico presuntivo de una colecistitis aguda. Los exámenes realizados revelaron una anemia microcítica hipocroma leve, recuento normal de leucocitos, eosinofilia absoluta de $2.189 / \mathrm{mm}^{3}$, plaquetas normales, y proteína $\mathrm{C}$ reactiva normal. Uremia, creatininemia, glicemia, proteinemia, calcemia, fosfemia, bilirrubinemia, amilasemia, transaminasa oxaloacética (GOT), fosfatasas alcalinas, colesterol y triglicéridos séricos normales. Transaminasa pirúvica (GPT) y gammaglucil-transferasa (GGT) leve- 
mente elevadas (59/81). El examen parasitológico de deposiciones y coprocultivo fueron negativos. Se realizó una ultrasonografía abdominal que mostró la vesícula biliar de paredes engrosadas con 4,5 $\mathrm{mm}$ de espesor en forma difusa, distendida, sin cálculos. La tomografía axial computada (TAC) de abdomen y pelvis efectuada concordó con los hallazgos descritos en la ecografía, describiéndose además una inflamación perivesicular y leve dilatación de la vía biliar intrahepática (Figura 1). Una colangio-resonancia confirmó los signos tomográficos y descartó un quiste de colédoco (Figura 2). Los síntomas y signos clínicos más los elementos sonográficos descritos se consideraron compatibles con el diagnóstico de una colecistitis aguda alitiásica. Buscando alguna etiología infecciosa se solicitaron serologías: IgM para virus de Epstein Barr, hepatitis A, Bartonella henselae, toxoplasmosis, hidatidosis humana, Fasciola hepatica y triquinosis, todas negativas. La serología (IgM e IgG) para citomegalovirus fue positiva. Se realizó una endoscopia digestiva alta que informó una antropatía nodular leve; se tomaron tres muestras para reacción de polimerasa en cadena (RPC) para CMV en tejido gástrico con resultado negativo. Al mismo tiempo, la marcada eosinofilia hizo plantear la sospecha de toxocarosis, la que se confirmó con IgG por ELISA (índice CO/DO 1,5) y Western blot (LDBIO Diagnostics) positivo para Toxocara canis-catis, efectuados en el centro de referencia nacional, Instituto de Salud Pública (ISP). El paciente se trató con ranitidina (3 $\mathrm{mg} / \mathrm{kg}$ al día) evolucionando con disminución del dolor y cese de náuseas; sin embargo, al día siguiente del alta, a los cinco días de observación, reingresó con iguales síntomas. Confirmada la infección por Toxocara spp., se trató con albendazol $5 \mathrm{mg} / \mathrm{kg}$ al día, por 15 días, con regresión total de los síntomas. Dos ecografías sucesivas mostraron desaparición de los hallazgos descritos en la primera ecografía. Los controles ambulatorios mensuales o bimensuales posteriores revelaron reaparición de los síntomas asociados a valores oscilantes de eosinofilia $\left(1.834,448,833 / \mathrm{mm}^{3}\right)$, por lo que repitió el tratamiento con buena respuesta clínica, siendo el último valor de 247 eosinófilos $/ \mathrm{mm}^{3}$.

\section{Discusión}

La toxocarosis es una zoonosis causada por larvas de los nemátodos del género Toxocara. Es una geohelmintiasis de perros y gatos capaces de infectar y enfermar accidentalmente al hombre, en particular a los niños ${ }^{1} \mathrm{y}$ por lo tanto importante para la salud pública, aunque se la considera menospreciada a nivel mundial. En Chile se han publicado estudios respecto de la caracterización demográfica de perros en Santiago ${ }^{2}$ y Viña del Mar ${ }^{3}$, así como reportes sobre el riesgo de infección por T. canis en casas con perros y lugares públicos de varias ciudades de Chile, incluyendo Valdivia ${ }^{4-6}$. También se han efectuado estudios de seroprevalencia en humanos ${ }^{7}$.

La toxocarosis humana o granulomatosis parasitaria es una parasitosis larval sistémica que se presenta en forma asintomática o con diversas manifestaciones como compromiso respiratorio, eosinofilia, fiebre, hepatomegalia, esplenomegalia, adenopatías, afectación del sistema nervioso central, ocular, miocardio y piel, pudiendo ser grave e incluso mortal ${ }^{1}$. Los niños se infectan al ingerir

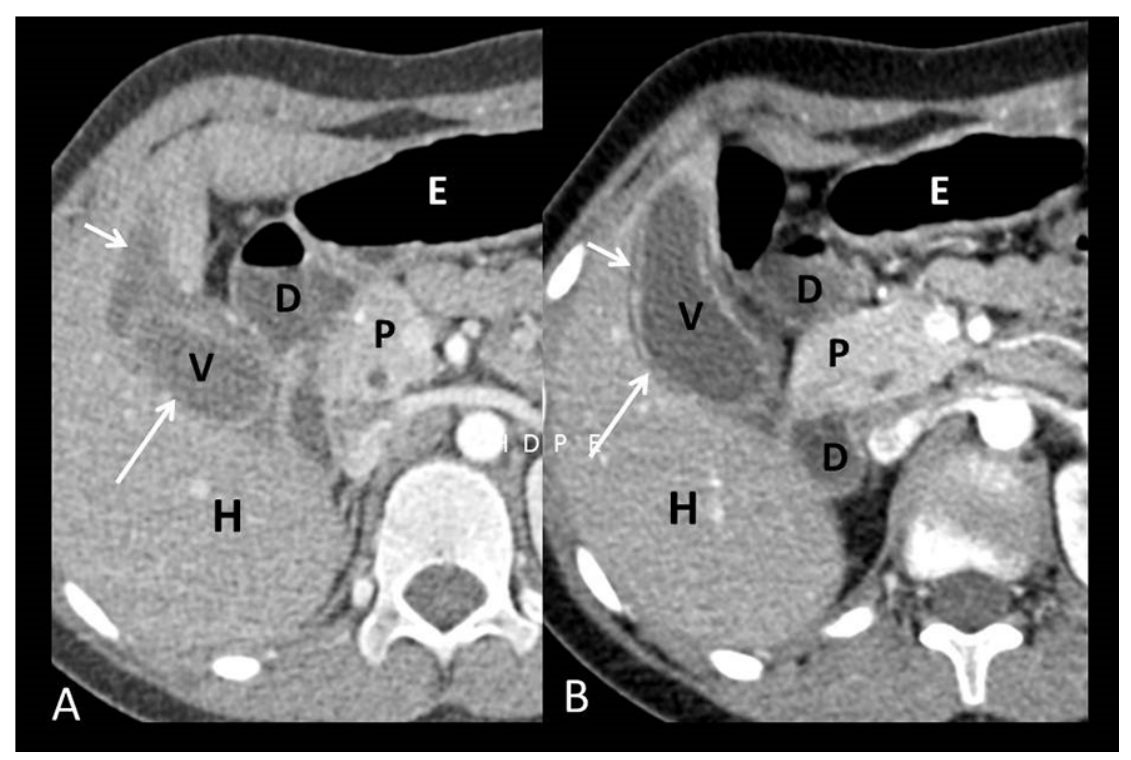

Figura 1 (A, B). Tomografía computada de abdomen con cortes axiales del hipocondrio derecho. Se identifica la vesícula biliar (V) distendida con contenido denso y engrosamiento de su pared (flechas largas) y signos de edema perivesicular (flechas cortas). H: hígado; D: duodeno; P: páncreas; E: estómago.

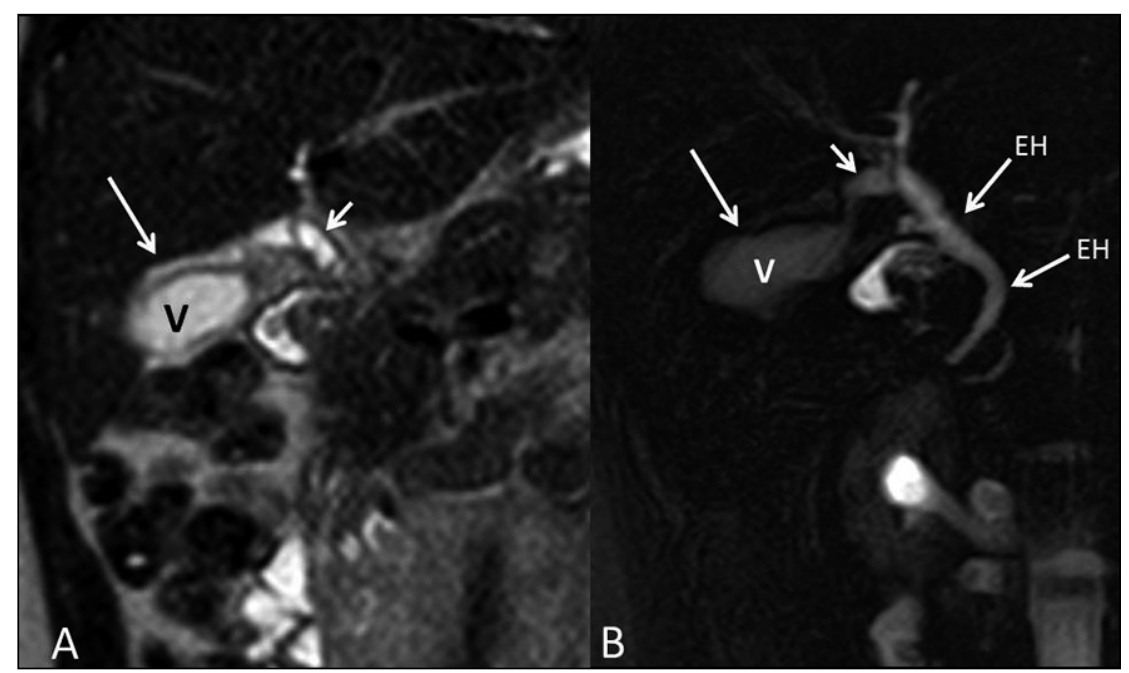

Figura 2. Resonancia magnética de abdomen con cortes coronales del hipocondrio derecho en secuencia ponderada en T2 (A) y colangiografía (B) muestran la vesícula biliar (V) distendida con engrosamiento de su pared y signos de edema perivesicular (flechas largas), el conducto cístico (flechas cortas) y la vía biliar extrahepática $(E H)$. 
huevos embrionados de T. canis a través de las manos contaminadas, ya sea por contacto directo con sus mascotas caninas en especial cachorros, a través del pelaje del perro ${ }^{6}$, o ingestión de vegetales o tierra contaminada con heces caninas ${ }^{8}$, en domicilio o en plazas y parques públicos ${ }^{5,6,8-14}$. Los factores de riesgo son las malas condiciones sanitarias e higiénicas, jugar en el suelo, comerse las uñas y la geofagia, así como el contacto con perros en el propio domicilio o callejeros ${ }^{13-15}$. Los síndromes clínicos de toxocarosis descritos (larva migrans visceral sistémica, larva migrans ocular, larva migrans neurológica, larva migrans encubierta y asintomática), así como el compromiso visceral aislado de algunos órganos como dermis, corazón, hígado, pulmón y músculo, son efecto de la migración de las larvas por vía sanguínea y la respuesta inmune del hospedero ${ }^{15}$. Las larvas secretan o excretan lectinas, mucinas y enzimas y tienen una cubierta rica en mucina que la larva abandona cuando es cubierta por anticuerpos y células de la respuesta inmune, dando lugar a una respuesta inflamatoria eosinofílica, pudiendo formar granulomas eosinofílicos en el sitio de localización ${ }^{16,17}$. Las manifestaciones clínicas y el curso de la enfermedad dependen de la cantidad del inóculo, la frecuencia de reinfecciones, la localización de la larva migrante y la respuesta del hospedero ${ }^{15,17}$. A pesar de las altas cifras de incidencia y prevalencia serológica notificadas, la publicación de casos clínicos pediátricos en cualquiera de sus formas es escasa, lo que probablemente refleja la subestimación de esta parasitosis. La sospecha y confirmación de toxocarosis humana por $T$. canis implica el reconocimiento de signos y síntomas clínicos compatibles con alguna de las manifestaciones clínicas reportadas, eosinofilia sanguínea, antecedente epidemiológico de contacto con perros o heces caninas y la demostración serológica por ELISA y Western blot ${ }^{15-17}$, ya que los parásitos no son detectables en el examen parasitológico directo de deposiciones. En nuestro paciente existía el antecedente de contacto con un perro en el propio domicilio y con alta probabilidad con sus desechos, síntomas clínicos compatibles con larva migrans visceral hepática y de vesícula biliar, eosinofilia sanguínea en ausencia de otra parasitosis o de alergia cutánea o respiratoria y demostración serológica por exámenes de referencia, ELISA y Western blot.

En Chile encontramos tres comunicaciones de series de casos en niños de toxocarosis. Noemí y cols. ${ }^{18}$, reportaron 42 niños entre 1979-1983, con evidencia clínica y serológica de infección por larva migrans visceral, con eosinofilia mayor a 500 cél $/ \mathrm{mm}^{3}, 64 \%$ con compromiso pulmonar y $59 \%$ hepatomegalia, encontrando anormalidades en el fondo de ojo en $4,8 \%$. Sánchez y cols. ${ }^{19}$, en un análisis retrospectivo, investigaron la afectación ocular en una cohorte de 175 niños de la misma zona geográfica, pero 28 años después, seropositivos para T. canis, a los que se les realizó una evaluación oftalmológica, encontrando 0,5\% (un niño) con hallazgos oftalmológicos (disminución de la visión del ojo afectado) compatibles con toxocarosis ocular. Cruces ${ }^{20}$ presentó un caso clínico radiológico de un lactante de sexo masculino con anemia, adenopatías cervicales y hepatomegalia, en el que se encontró leucocitosis en rango leucemoide $\left(75.700\right.$ céls $\left./ \mathrm{mm}^{3}\right)$ con $69 \%$ de eosinófilos (eosinofilia absoluta mayor a 50.000 céls/ $\mathrm{mm}^{3}$ ) e IgG positiva para T. canis.

Aunque desde el punto de vista teórico es posible la inflamación y formación de granulomas eosinofílicos en la vesícula biliar, que ocasionen obstrucción de ella, no encontramos en casos pediátricos de otros países, algún caso de toxocarosis con síntomas clínicos de colecistitis aguda. Altcheh ${ }^{21}$ comunicó los aspectos clínicos y de laboratorio de 54 pacientes, reafirmando que la mayoría de los infectados eran asintomáticos y los sintomáticos presentaron los síndromes clínicos previamente señalados, incluyendo un caso con miocarditis. Ballón ${ }^{22}$ notificó cuatro casos de manifestaciones no sospechadas de toxocarosis en niños (convulsión febril y alteraciones sensoriales calificadas como meningoencefalitis aguda, asma bronquial, dermatitis migratoria y hepatomegalia asociada a fiebre intermitente). Martin ${ }^{23}$, en Argentina, reportó los hallazgos clínicos y serológicos en 100 niños con diagnóstico presuntivo de toxocarosis destacando la eosinofilia como un elemento de apoyo diagnóstico, presente en todos los infectados. $\mathrm{Li}^{24}$ efectuó una revisión sistemática, confirmando la asociación entre asma y toxocarosis en niños. Por último, Carvalho ${ }^{25}$, en una extensa revisión bibliográfica (1952-2009) sobre toxocarosis en niños, detalla los factores de riesgo, presentación clínica, exámenes de laboratorio y de imágenes que pueden apoyar el diagnóstico, corroborando lo descrito anteriormente: sugiere entonces estudios prospectivos para garantizar el acceso al mejor tratamiento y recomendando la prevención ${ }^{25}$. Rubinsky ${ }^{26}$ y Schmidt ${ }^{27}$ hicieron una revisión de los nuevos avances en diagnóstico, tratamiento, seguimiento e intervenciones en base a marcadores inmunológicos avanzados que podrían estar accesibles en un futuro próximo.

Las enfermedades de la vesícula biliar son infrecuentes en niños. Por cada 1.000 casos de adultos existen 1,3 casos pediátricos. La CA es una enfermedad inflamatoria aguda de la vesícula biliar, a menudo atribuida a cálculos aunque muchos otros factores como isquemia, alteraciones de la motilidad, injuria química directa, infecciones, incluyendo protozoos y otros parásitos, enfermedades del colágeno y reacciones alérgicas, están involucrados. En adultos, la litiasis explica $85-90 \%$ de todas las causas de $\mathrm{CA}$, mientras que la colecistitis aguda acalculosa (CAA) explica el restante $5-15 \%{ }^{28}$. La CAA es una enfermedad muy infrecuente en niños. Tsakayannis ${ }^{28}$ reportó una frecuencia de $30-50 \%$ del total de casos de CA, com- 
parado con $2-17 \%$ en adultos. Puede presentarse en dos formas distintas: aguda (duración de síntomas menos de un mes) y crónica (duración más de tres meses). Barie ${ }^{29}$ publicó una detallada y completa descripción de CAA, en adultos y niños, tanto en los aspectos clínicos, factores patogénicos, métodos de diagnóstico y complicaciones, enfatizando la utilidad de los criterios imagenológicos. El diagnóstico de CAA en niños no es fácil, puede sospecharse clínicamente como una CA, pero sólo se confirma con exámenes imagenológicos y eventualmente histológicos si la terapia considera una colecistectomía. La ecografía o ultrasonografía de la vesícula biliar ha sido considerada una herramienta diagnóstica segura y efectiva, de fácil accesibilidad incluso en los servicios de pacientes críticos y de emergencia ${ }^{30}$. Los criterios ecográficos de CAA aceptados en niños son: el grosor de pared vesicular de $3 \mathrm{~mm}$ o más, el diámetro del conducto biliar común de $3 \mathrm{~mm}$ o más, ausencia de cálculos, presencia de líquido pericolecistítico y signo sonográfico de Murphy ${ }^{30}$. Varias entidades pueden evidenciarse sonográficamente como una pared vesicular engrosada, tales como barro biliar, pequeños cálculos que no dan sombra, colesterolosis y ascitis; sin embargo, excluyendo estas entidades se ha considerado que el grosor de la pared de la vesícula biliar es un criterio diagnóstico confiable y específico de CAA. Tsai ${ }^{31}$ en un estudio retrospectivo, concluyó que los hallazgos del ultrasonido en colecistitis pediátrica tiene menor sensibilidad y valor predictor positivo que en los adultos, lo que podría explicarse por la menor prevalencia de colecistitis crónica en niños, comparados con los adultos, episodios más leves de inflamación autolimitada de la vesícula biliar, lo que podría llevar a un retraso en el tratamiento; sin embargo, el mismo autor comenta que los reportes de adultos incluían pacientes con CA, en tanto que en su estudio, $98 \%$ de los pacientes se presentaba como dolor abdominal, y no como CA. Lee planteó algunas dudas sobre el significado de los hallazgos imagenológicos considerados como criterio para el diagnóstico de CAA. Al analizar en forma retrospectiva 67 niños con engrosamiento de la pared vesicular acalculosa, encontró un amplio rango de condiciones que no son colecistitis que causan engrosamiento difuso de la vesícula biliar, que se resuelven espontáneamente o con el tratamiento de la enfermedad subyacente. Recomienda incluso evitar los tratamientos quirúrgicos si no hay manifestaciones clínicas definitivas de colecistitis. Las etiologías encontradas por Lee fueron: infección sistémica en 24 pacientes (bacterias, virus [Epstein Barr y parvovirus] y hongos), hepatitis aguda en 18, enfermedades sistémicas en 11 (LES, hipersensibilidad a fármacos, insuficiencia cardíaca congestiva, enfermedad renal, síndrome nefrótico, insuficiencia renal aguda, SDRA, linfangiectasia intestinal y asfixia), linfohistiocitosis hemofagocítica en cuatro, pancreatitis aguda en tres, hepatopatías específicas en otros tres y misceláneos en cuatro. Punia ${ }^{32}$ analizó el espectro clínico- histopatológico de la enfermedad de la vesícula biliar en niños, encontrando en 56 colecistectomías, 7\% quiste de colédoco y $21 \%$ de CAA sin precisar la probable etiología de ella.

La CAA se ha asociado a mal pronóstico por el retraso en el diagnóstico y tratamiento, así como a la presentación en pacientes graves o con co-morbilidades ${ }^{33-36}$. De las publicaciones disponibles, es evidente que la CAA puede presentarse en niños aparentemente $\operatorname{sanos}^{37}$, en pacientes con patologías médicas ${ }^{38-40}$ y quirúrgicas ${ }^{41}$, en infecciones graves de distinta etiología ${ }^{42-44}$, como también en infecciones banales ${ }^{45}$. En el caso de las infecciones virales por CMV y virus de Epstein Barr se presentan en general en pacientes con algún grado de inmunodeficiencia previa, aunque también se han descrito en sujetos inmunocompetentes ${ }^{46-48}$. En nuestro paciente la etiología viral investigada puede considerarse negativa ya que los anticuerpos positivos para CMV no son lo suficientemente específicos para atribuirles un rol etiológico $^{49}$ y la RPC para CMV fue negativa. Las infecciones bacterianas se producen en general en el contexto de un paciente grave, aunque hay casos descritos de CAA en pacientes con fiebre tifoidea, en que sólo los más graves requirieron colecistectomía. En relación a las infecciones parasitarias y por protozoos, se han reportado CAA por Plasmodium falciparum ${ }^{50}$ y Ascaris lumbricoides ${ }^{51}$ los que se han recuperado con el tratamiento médico específico y ocasionalmente con cirugía. Por lo tanto, parece ser que la opción de tratamiento de la CAA está relacionada más bien con la gravedad del paciente y la posibilidad de respuesta a tratamiento médico, reservando la cirugía para los casos de mala evolución, en muchos de los cuales se ha constatado histológicamente una colecistitis acalculosa gangrenosa de mal pronóstico. Lo particular de nuestro caso es la manifestación clínica de toxocarosis como CAA, pues el paciente ingresó con el diagnóstico presuntivo de CA, y los exámenes de apoyo diagnóstico por tres técnicas: sonografía, tomografía computada de vía biliar y colangioresonancia, confirmaron la inflamación de la vesícula biliar con ausencia de cálculos, que regresó completamente después del tratamiento con albendazol, al igual que los síntomas clínicos.

La CAA por larva migrans visceral ha sido escasamente reportada en el mundo. Kaki y Yoshiji reportaron un caso de colecistitis eosinofílica junto con pericarditis y derrame pleural, atribuida inicialmente a Ascaris lumbricoides $^{51}$, y luego a larva migrans visceral ${ }^{52}$ en una mujer joven, sin antecedentes de alergia, que consultó por dolor en hipocondrio derecho, disnea y erupción cutánea, en la cual los exámenes imagenológicos (ultrasonografía y colangioresonancia) demostraron un marcado aumento de volumen y grosor de la pared vesicular, sin cálculos, en tanto la TAC de tórax y ecocardiografía revelaron 
derrame pleural y pericárdico; asociado a una acentuada eosinofilia ( $31 \%$ de 7.500 leucocitos) con elevación de IgE total $(246.6 \mathrm{u} / \mathrm{ml})$ y títulos de anticuerpos positivos contra Ascaris suum y anticuerpos a T. canis 100-150 veces mayor que el control normal. El tratamiento con albendazol mejoró las manifestaciones clínicas junto con la normalización de los hallazgos imagenológicos, eosinofilia e IgE por lo que no fue necesaria la colecistectomía, por lo que no hubo confirmación histológica de la colecistitis eosinofílica. Montiel-Jarquín ${ }^{53}$ objetó la calificación de este caso como una colecistitis eosinofílica. Sugirió además que la migración del parásito, A. lumbricoide, fue demostrado sólo por serología, lo que no confirma la presencia del parásito en el momento de la colecistitis, ya que los anticuerpos pueden estar positivos sin que el parásito esté presente en el organismo; y que tal vez la infección por T. canis demostrado por ELISA, sea la causa de CA y no la infiltración eosinofílica de la vesícula, que sólo podría demostrarse con el examen histológico de la vesícula biliar. Choo ${ }^{54}$ reportó el caso de una mujer con CAA por T. canis confirmada, la que podría ser el segundo caso de un adulto con esta asociación. Por último, la CAA eosinofílica, es una entidad muy infrecuente descrita en 1949, asociada a variadas causas de esosinofilia tales como: alergias, gastroenteritis eosinofílica, síndrome hipereosinofílico, síndrome eosinofilia-mialgia, medicamentos de hierbas e incluso parásitos, cuyo diagnóstico se realiza a través de la histología de la vesícula biliar, considerándose más que un cuadro específico, sólo una forma de CA, la mayor parte de las veces acalculosa. Del Moral comunicó el caso de una mujer joven con CA eosinofílica acalculosa confirmada por histología, con recuento normal de eosinófilos en sangre, en la cual no se encontró una causa identificable por lo que la catalogó de idiopática, aunque no se menciona la realización de exá- menes directos o serológicos para investigar parásitos ${ }^{55}$.

En resumen, la manifestación clínica de nuestro paciente como CAA, sin tener la confirmación histológica de colecistitis aguda eosinofílica o no eosinofílica, puede corresponder a una presentación clínica de toxocarosis no reconocida o poco reconocida previamente, o a un cuadro de larva migrans visceral de manifestación preferentemente hepatobiliar, sin hepatomegalia, en la cual los hallazgos vesiculares encontrados son sólo elementos fisiológicos en el contexto de larva migrans sistémica, los que cedieron con el tratamiento de la enfermedad de base.

Agradecimientos: Los autores agradecen a Cristián García B. por la asesoría en la elección e interpretación de las imágenes y la revisión del caso clínico y a Felipe Dueñas por la excelente colaboración bibliográfica y preparación del manuscrito.

\section{Resumen}

La toxocarosis humana es una parasitosis larvaria crónica catalogada dentro de las cinco enfermedades subestimadas más importantes por los CDC. Las larvas pueden diseminarse por vía sistémica y migrar a distintos tejidos (larva migrans visceral) incluyendo el hígado y vesícula biliar. La colecistitis aguda acalculosa (CAA) es una enfermedad rara en niños. El diagnóstico se basa en parámetros clínicos y criterios imagenológicos. Se ha asociado a sepsis, shock, trauma, quemaduras, enfermedades sistémicas graves, anomalías congénitas e infecciones, como también en niños sanos. Presentamos el caso de una toxocarosis infantil con síntomas clínicos y criterios imagenológicos compatibles con una CAA tratado médicamente, y discutir la relación entre ambos cuadros en base a la evidencia publicada.

\section{Referencias bibliográficas}

1.- Archelli S, Kozubsky L. Toxocara y toxocariosis. Acta Bioquim Clin Latinoam 2008; 42: 379-84.

2.- Ibarra L, Morales M, Acuña P. Aspectos demográficos de la población de perros y gatos en la ciudad de Santiago. Chile. Avances en Ciencias Veterinarias 2003; 18: 13-20.

3.- Morales M, Varas C, Ibarra L. Caracterización demográfica de la población de perros en Viña del Mar. Chile. Arch Med Vet 2009; 41: 89-95.

4.- Pavletic C. El Control de las Poblaciones Caninas en los Centros Urbanos. Una Visión de Salud Pública [Internet]. Ministerio de Salud; 2011. Disponible en: http://www.munitel.cl/ eventos/seminarios $/ \mathrm{html} /$ documentos $/ 2010 /$ SEMINARIO POSTURA MUNICIPAL POR_ANIMALES_POTENCIALMENTE_
PELIGROSOS/PPT07.pdf (accedido el 4 de septiembre de 2015)

5.- Reyes Y. Determinación del riesgo de infección con huevos de Toxocara canis en lugares públicos y patios de casas particulares en la ciudad de Valdivia, Chile. Memoria de título, 2008, Universidad Austral de Chile. Valdivia. Disponible en: cybertesis.uach.cl/tesis/ uach/2008/fvr457d/doc/fvr457d.pdf (accedido el 4 de septiembre de 2015).

6.- Lorca M, Eberlein C, Molina P, Cortés M, Escobar S. Comparación de la frecuencia de huevos de Toxocara canis en pelaje y fecas de caninos con propietario conocido en la ciudad de Viña del Mar [Internet]. Décimas Jornadas Científicas Instituto de Salud Pública: 2011. Disponible en: http://www.ispch.cl/documentos/ libro xjc v2012.pdf (accedido el 4 de septiembre de 2015).
7.- Navarrete N, Rojas E. Seroprevalencia de Toxocarosis en donantes de sangre. Arch Med Vet 1998; 30: 153-6.

8.- Archelli S, Santillan G I, Fonrouge R, Céspedes G, Burgos L, Radman N. Toxocariasis: seroprevalence in abandoned-institutionalized children and infants. Rev Argent Microbiol 2014; 46: 3-6.

9.- $\quad$ Fu C J, Chuang T W, Lin H S, Wu C H, Liu Y C, Langinlur M K, et al. Seroepidemiology of Toxocara canis infection among primary school children in the capital area of the Republic of the Marshall Islands. BMC Infect Dis 2014; 14: 261.

10.- Manini M, Marchioro A A, Colli C M, Nishi L, Falavigna-Guilherme A L. Association between contamination of public squares and seropositivity for Toxocara spp. in children. Vet Parasitol 2012; 188: 48-52. 
11.- Maraghi S, Rafiei A, Hajihossein R, Sadjjadi S. Seroprevalence of toxocariasis in hypereosinophilic individuals in Ahwaz, southwestern Iran. J Helminthol 2012; 86: 241-4.

12.- Kanobana K, Devleesschauwer B, Polman K, Speybroeck N. An agent- based model of exposure to human toxocariasis: a multi-country validation. Parasitology 2013; 140: 986-98.

13.- Romero C, Mendoza G, Yáñez S, Ponce M, Bustamante P, Ramírez N. Prevalence and risk factors associated with Toxocara canis infection in children. Sci World J 2013; 2013: 572089.

14.- Santarém V A, Leli F N, Rubinsky-Elefant G, Giuffrida R. Protective and risk factors for toxocariasis in children from two different social classes of Brazil. Rev Inst Med Trop Sao Paulo 2011; 53: 61-5.

15.- Roldán W H, Espinoza Y A, Huapaya P E, Jiménez S. Diagnóstico de la toxocariasis humana. Rev Peru Med Exp Salud Publica 2010; 27: 613-20.

16.- Maizels R M. Toxocara canis: molecular basis of immune recognition and evasion. Vet Parasitol 2013; 193: 365-74

17.- Macpherson $\mathrm{C}$ N. The epidemiology and public health importance of toxocariasis: a zoonosis of global importance. Int J Parasitol 2013; 43: 999-1008.

18.- Noemí H, Schuh W, Herskovic P, Ríos E, Cerva $\mathrm{L}$, Torres $\mathrm{M}$, et al. Larva migrans visceral en niños. Rev Chil Pediatr 1984; 55: 244-8.

19.- Sánchez J, López J, González M, Villaseca E, Manieu D, Roizen B, et al. Detección de lesiones oculares en niños seropositivos para Toxocara canis. Rev Chilena Infect 2011; 28: 431-4.

20.- Cruces P, Donoso A, Cruces A, Cruces K. Caso clínico-radiológico para diagnóstico. Rev Chil Pediatr 2005; 76: 411-43.

21.- Altcheh J, Nallar M, Conca M, Biancardi M, Freilij H. Toxocariasis: aspectos clínicos y de laboratorio en 54 pacientes. An Pediatr (Barc) 2003; 58: 425-31.

22.- Ballón A, Chalco J, Candela J. Manifestaciones no sospechadas de toxocariasis en niños. Discusión a propósito de 4 casos. Rev Peruana Ped 2004; 46-50.

23.- Martin U, Machuca P, Demonte M, Contini L. Estudio en niños con diagnóstico presuntivo de toxocariasis en Santa Fé, Argentina. Medicina (B. Aires) 2008; 68: 353-7.

24.- Li L, Gao W, Yang X, Wu D, Bi H, Zhang S, et al. Asthma and toxocariasis. Ann Allergy Asthma Immunol 2014; 113: 187-92.

25.- Carvalho E A, Rocha R L. Toxocariasis: visceral larva migrans in children. J Pediatr (Rio J) 2011; 87: 100-10.

26.- Rubinsky-Elefant G, Hoshino-Schimizu S, Jacob C M, Sánchez M C, Ferreira A W. Potential immunological markers for diagnosis and therapeutic assessment of toxocariasis. Rev
Inst Med Trop Sao Paulo 2011; 53: 61-5.

27.- Moreira G, Telmo P, Mendonça M, Moreira A, McBride A, Scaini C, et al. Human toxocariasis: current advances in diagnostics, treatment, and interventions. Trends Parasitol 2014; 30: 45664.

28.- Tsakayannis D E, Kozakewich H P, Lillehei C W. Acalculous cholecystitis in children. J Pediatr Surg 1996; 31: 127-30.

29.- Barie P C, Eachempati S R. Acute acalculous cholecystitis. Curr Gastroenterol Rep 2003; 5: 302-9.

30.- Tsung J W, Raio C C, Ramírez-Schrempp D, Blaivas M. Point-of-care ultrasound diagnosis of pediatric cholecystitis in the ED. Am J Emerg Med 2010: 28; 338-42.

31.- Tsai J, Sulkowski J P, Cooper J N, Mattei P, Deans K J, Minneci P C. Sensitivity and predictive value of ultrasound in pediatric cholecystitis. J Surg Res 2013; 184: 378-82.

32.- Lee J H, No Y E, Lee Y J, Hwang J Y, Lee J W, Park J H. Acalculous diffuse gallbladder wall thickening in children. Pediatr Gastroenterol Hepatol Nutr 2014; 17: 98-103.

33.- Punia R P, Garg S, Bisht B, Dalal U, Mohan H. Clínico-pathological spectrum of gallbladder disease in children. Acta Paediatr 2010; 99 : 1561-4.

34.- Nikfarjam M, Manya K, Fink M A, Hadj A K, Muralidharan V, Starkey G, et al. Outcomes of patients with histologically proven acute acalculous cholecystitis. ANZ J Surg 2012; 82: 918-22.

35.- Karkera P J, Sandlas G, Ranjan R, Gupta A, Kothari P. Acute acalculous cholecystitis causing gall bladder perforation in children. $\mathrm{J}$ Indian Assoc Pediatr Surg 2010; 15: 139-41.

36.- Huang S C, Yang Y C. Septic shock and hypofibrinogenemia predict a fatal outcome in childhood acute acalculous cholecystitis. J Pediatr Gastroenterol Nutr 2011; 53: 548-52.

37.- McNaughton C, Morrow S, Cico S J. Acalculous cholecystitis in a healthy 4-year-old boy. Am J Emerg Med 2011; 29: 962.e1-2.

38.- Oksenberg T, Urra F, Nilsen E. Colecistitis aguda alitiásica. Rev Chil Pediatr 1996; 67: 1279.

39.- Shihabuddin B, Sivitz A. Acute acalculous cholecystitis in a 10-year-old girl with cystic fibrosis. Pediatr Emerg Care 2013; 29: 117-21.

40.- Sanches B F, Martins T, Santos M J, Azeredo P. Acute acalculous cholecystitis in a patient with juvenile dermatomyositis. BMJ Case Rep 2014; 2014. pii: bcr2014205066.

41.- Özkaya AK, Güler E, Cetinkaya A, Karakaya A E, Göksügür Y, Katı Ö, et al. HenochSchöenlein purpura complicated by acalculous cholecystitis and intussusception, and following recurrent appendicitis. Pediatr Int Child Health 2015: 2046905515Y0000000019.

42.- Gnassingbé K, Katakoa G, Kanassoua K K,
Adabra K, Mama W, Simlawo K, et al. Acute cholecystitis from typhic origin in children. Afr J Pediatr Surg 2013; 10: 108-11.

43.- Herek O, Cördük N, Herek D, Bagci S. Acute acalculous cholecystitis due to hepatitis A infection in a child: a rare cause of acute abdomen. Ann Afr Med 2011; 10: 193-5.

44.- Prashanth G P, Angadi B H, Joshi S N, Bagalkot P S, Maralihalli M B. Unusual cause of abdominal pain in pediatric emergency medicine. Pediatr Emerg Care 2012; 28: 560-1.

45.- Gora-Gebka M, Liberek A, Bako W, Szarszewski A, Kaminska B, Korzon M. Acute acalculous cholecystitis of viral etiology-a rare condition in children? J Pediatr Surg 2008; 43: e25-7.

46.- Kim A, Yang H R, Moon J S, Chang J Y, Ko J S. Epstein-Barr virus infection with acute acalculous cholecystitis. Pediatr Gastroenterol Hepatol Nutr 2014; 17: 57-60.

47.- Alkhoury F, Díaz D, Hidalgo J. Acute acalculous cholecystitis (ACC) in the pediatric population associated with Epstein-Barr virus (EBV) infection. Case report and the review of the literature. Int J Surg Case Rep 2015; 11: 502.

48.- Pawlowska A. Mroczkowska S, Golyska D, Kominek K, Pac E. Acute acalculous cholecystitis in a 17-year-old girl with EpsteinBarr virus infection. Prz Gastroenterol 2015; 10: 54-6.

49.- Griffiths P, Baraniak I, Reeves M. The pathogenesis of human cytomegalovirus. J Pathol 2015; 235: 288-97.

50.- Kuttiat V S, Kohli U. Acute acalculous cholecystitis associated with malarial infection in children: report of two cases. J Trop Pediatr 2006; 53: 59-61.

51.- Kaji K, Yoshiji H, Yoshikawa M, Yamazaki M, Ikenaka Y, Noguchi R, et al. Eosinophilic cholecystitis along with pericarditis caused by Ascaris lumbricoides: a case report. World J Gastroenterol 2007; 13: 3760-2.

52.- Yoshiji H, Yoshikawa M, Kaji K, Fukui H. Eosinophilic cholecystitis as a rare manifestation of visceral larva migrans. World J Gastroenterol 2007; 13: 6119.

53.- Montiel-Jarquín A. Eosinophilic cholecystitis caused by Ascaris lumbricoides. World J Gastroenterol 2008; 14: 2783.

54.- Choo J W, Choi H J, Moon J H, Chung J H, Park J S, Myung Y S, et al. A case of acute cholecystitis combined with eosinophilic pericarditis caused by toxocariasis. Korean $\mathrm{J}$ Med 2013; 84: 836-41.

55.- Del-Moral-Martínez M, Barrientos-Delgado A, Crespo-Lara V, Cervilla-Sáez-de- Tejada M E, Salmerón-Escobar J. Eosinophilic cholecystitis: An infrecuent cause of acute cholecystitis. Rev Esp Enferm Dig 2015; 107: 45-7. 